\title{
COMMON FIXED POINT AND INVARIANT APPROXIMATION RESULTS IN CERTAIN METRIZABLE TOPOLOGICAL VECTOR SPACES
}

\author{
NAWAB HUSSAIN AND VASILE BERINDE
}

Received 27 June 2005; Revised 1 September 2005; Accepted 6 September 2005

We obtain common fixed point results for generalized $I$-nonexpansive $R$-subweakly commuting maps on nonstarshaped domain. As applications, we establish noncommutative versions of various best approximation results for this class of maps in certain metrizable topological vector spaces.

Copyright (C) 2006 N. Hussain and V. Berinde. This is an open access article distributed under the Creative Commons Attribution License, which permits unrestricted use, distribution, and reproduction in any medium, provided the original work is properly cited.

\section{Introduction and preliminaries}

Let $X$ be a linear space. A $p$-norm on $X$ is a real-valued function on $X$ with $0<p \leq 1$, satisfying the following conditions:

(i) $\|x\|_{p} \geq 0$ and $\|x\|_{p}=0 \Leftrightarrow x=0$,

(ii) $\|\alpha x\|_{p}=|\alpha|^{p}\|x\|_{p}$,

(iii) $\|x+y\|_{p} \leq\|x\|_{p}+\|y\|_{p}$

for all $x, y \in X$ and all scalars $\alpha$. The pair $\left(X,\|,\|_{p}\right)$ is called a $p$-normed space. It is a metric linear space with a translation invariant metric $d_{p}$ defined by $d_{p}(x, y)=\|x-y\|_{p}$ for all $x, y \in X$. If $p=1$, we obtain the concept of the usual normed space. It is wellknown that the topology of every Hausdorff locally bounded topological linear space is given by some $p$-norm, $0<p \leq 1$ (see [9] and references therein). The spaces $l_{p}$ and $L_{p}$, $0<p \leq 1$ are $p$-normed spaces. A $p$-normed space is not necessarily a locally convex space. Recall that dual space $X^{*}$ (the dual of $X$ ) separates points of $X$ if for each nonzero $x \in X$, there exists $f \in X^{*}$ such that $f(x) \neq 0$. In this case the weak topology on $X$ is well-defined and is Hausdorff. Notice that if $X$ is not locally convex space, then $X^{*}$ need not separate the points of $X$. For example, if $X=L_{p}[0,1], 0<p<1$, then $X^{*}=\{0\}([12$, pages 36 and 37]). However, there are some non-locally convex spaces $X$ (such as the $p$-normed spaces $l_{p}, 0<p<1$ ) whose dual $X^{*}$ separates the points of $X$.

Let $X$ be a metric linear space and $M$ a nonempty subset of $X$. The set $P_{M}(u)=\{x \in$ $M: d(x, u)=\operatorname{dist}(u, M)\}$ is called the set of best approximants to $u \in X$ out of $M$, where $\operatorname{dist}(u, M)=\inf \{d(y, u): y \in M\}$. Let $f: M \rightarrow M$ be a mapping. A mapping $T: M \rightarrow M$ 
is called an $f$-contraction if there exists $0 \leq k<1$ such that $d(T x, T y) \leq k d(f x, f y)$ for any $x, y \in M$. If $k=1$, then $T$ is called $f$-nonexpansive. A mapping $T: M \rightarrow M$ is called condensing if for any bounded subset $B$ of $M$ with $\alpha(B)>0, \alpha(T(B))<\alpha(B)$, where $\alpha(B)=\inf \{r>0: B$ can be covered by a finite number of sets of diameter $\leq r\}$. A mapping $T: M \rightarrow M$ is hemicompact if any sequence $\left\{x_{n}\right\}$ in $M$ has a convergent subsequence whenever $d\left(x_{n}, T x_{n}\right) \rightarrow 0$ as $n \rightarrow \infty$. The set of fixed points of $T$ (resp. $f$ ) is denoted by $F(T)$ (resp. $F(f))$. A point $x \in M$ is a common fixed point of $f$ and $T$ if $x=f x=T x$. The pair $\{f, T\}$ is called (1) commuting if $T f x=f T x$ for all $x \in M$; (2) $R$-weakly commuting [16] if for all $x \in M$ there exists $R>0$ such that $d(f T x, T f x) \leq R d(f x, T x)$. If $R=1$, then the maps are called weakly commuting. The set $M$ is called $q$-starshaped with $q \in M$ if the segment $[q, x]=\{(1-k) q+k x: 0 \leq k \leq 1\}$ joining $q$ to $x$, is contained in $M$ for all $x \in M$. Suppose that $M$ is $q$-starshaped with $q \in F(f)$ and is both $T$ - and $f$-invariant. Then $T$ and $f$ are called $R$-subweakly commuting on $M$ (see [17]) if for all $x \in M$, there exists a real number $R>0$ such that $d(f T x, T f x) \leq R \operatorname{dist}(f x,[q, T x])$. It is well-known that commuting maps are $R$-subweakly commuting maps and $R$-subweakly commuting maps are $R$-weakly commuting but not conversely in general (see $[16,17])$.

A set $M$ is said to have property $(N)$ if $[7,11]$

(i) $T: M \rightarrow M$,

(ii) $\left(1-k_{n}\right) q+k_{n} T x \in M$, for some $q \in M$ and a fixed sequence of real numbers $k_{n}\left(0<k_{n}<1\right)$ converging to 1 and for each $x \in M$.

A mapping $f$ is said to have property $(C)$ on a set $M$ with property $(N)$ if $f\left(\left(1-k_{n}\right) q+\right.$ $\left.k_{n} T x\right)=\left(1-k_{n}\right) f q+k_{n} f T x$ for each $x \in M$ and $n \in N$.

We extend the concept of $R$-subweakly commuting maps to nonstarshaped domain in the following way (see [7]):

Let $f$ and $T$ be self-maps on the set $M$ having property $(N)$ with $q \in F(f)$. Then $f$ and $T$ are called $R$-subweakly commuting on $M$, provided for all $x \in M$, there exists a real number $R>0$ such that $d(f T x, T f x) \leq R d\left(f x, T_{n} x\right)$ where $T_{n} x=\left(1-k_{n}\right) q+k_{n} T x$, and the sequence $\left\{k_{n}\right\}$ is as in definition of property $(N)$ of $M$. Each $T$-invariant $q$-starshaped set has property $(N)$ but not conversely in general. Each affine map on a $q$-starshaped set $M$ satisfies condition $(C)$.

Example 1.1 [7]. Consider $X=R^{2}$ and $M=\{(0, y): y \in[-1,1]\} \cup\{(1-1 /(n+1), 0)$ : $n \in N\} \cup\{(1,0)\}$ with the metric induced by the norm $\|(a, b)\|=|a|+|b|,(a, b) \in R^{2}$. Define $T$ on $M$ as follows:

$$
T(0, y)=(0,-y), \quad T\left(1-\frac{1}{n+1}, 0\right)=\left(0,1-\frac{1}{n+1}\right), \quad T(1,0)=(0,1)
$$

Clearly, $M$ is not starshaped [11] but $M$ has the property $(N)$ for $q=(0,0)$ and $k_{n}=$ $1-1 /(n+1)$. Define $I(0, y)=I(1-1 /(n+1), 0)=(0,0), I(1,0)=(1,0)$. Then $\| T I x-$ $I T x \|=0$ or 1 . Thus for all $x$ in $M,\|T I x-I T x\| \leq R\left\|k_{n} T x-I x\right\|$ with each $R \geq 1$ and $q=(0,0) \in F(I)$. Thus $I$ and $T$ are $R$-subweakly commuting but not commuting on $M$.

The map $T: M \rightarrow X$ is said to be completely continuous if $\left\{x_{n}\right\}$ converges weakly to $x$ implies that $\left\{T x_{n}\right\}$ converges strongly to $T x$. 
In 1963, Meinardus [10] employed the Schauder fixed point theorem to prove a result regarding invariant approximation. In 1979, Singh [19] proved the following extension of "Meinardus" result.

Theorem 1.2. Let $T$ be a nonexpansive operator on a normed space $X, M$ be a T-invariant subset of $X$ and $u \in F(T)$. If $P_{M}(u)$ is nonempty compact and starshaped, then $P_{M}(u) \cap$ $F(T) \neq \varnothing$.

In 1988, Sahab et al. [13] established the following result which contains Theorem 1.2 and many others.

Theorem 1.3. Let $I$ and $T$ be selfmaps of a normed space $X$ with $u \in F(I) \cap F(T), M \subset$ $X$ with $T(\partial M) \subset M$, and $q \in F(I)$. If $P_{M}(u)$ is compact and q-starshaped, $I\left(P_{M}(u)\right)=$ $P_{M}(u), I$ is continuous and linear on $P_{M}(u), I$ and $T$ are commuting on $P_{M}(u)$ and $T$ is $I$-nonexpansive on $P_{M}(u) \cup\{u\}$, then $P_{M}(u) \cap F(T) \cap F(I) \neq \varnothing$.

Let $D=P_{M}(u) \cap C_{M}^{I}(u)$, where $C_{M}^{I}(u)=\left\{x \in M: I x \in P_{M}(u)\right\}$.

Theorem 1.4 [1, Theorem 3.2]. Let $I$ and $T$ be selfmaps of a Banach space $X$ with $u \in$ $F(I) \cap F(T), M \subset X$ with $T(\partial M \cap M) \subset M$. Suppose that $D$ is closed and q-starshaped with $q \in F(I), I(D)=D, I$ is linear and continuous on $D$. If I and $T$ are commuting on $D$ and $T$ is $I$-nonexpansive on $D \cup\{u\}$ with $\operatorname{cl}(T(D))$ compact, then $P_{M}(u) \cap F(T) \cap F(I) \neq \varnothing$.

Recently, by introducing the concept of non-commuting maps to this area, Shahzad [14-18], Hussain and Khan [6] and Hussain et al. [7], further extended and improved the above mentioned results to non-commuting maps.

The aim of this paper is to prove new results extending and subsuming the above mentioned invariant approximation results. To do this, we establish a general common fixed point theorem for $R$-subweakly commuting generalized $I$-nonexpansive maps on nonstarshaped domain in the setting of locally bounded topological vector spaces, locally convex topological vector spaces and metric linear spaces. We apply a new theorem to derive some results on the existence of best approximations. Our results unify and extend the results of Al-Thagafi [1], Dotson [3], Guseman and Peters [4], Habiniak [5], Hussain and Khan [6], Hussain et al. [7], Khan and Khan [9], Sahab et al. [13], Shahzad [14-18], and Singh [19].

\section{Common fixed point and approximation results}

The following common fixed point result is a consequence of Theorem 1 of Berinde [2], which will be needed in the sequel.

Theorem 2.1. Let $M$ be a closed subset of a metric space $(X, d)$ and $T$ and $f$ be $R$-weakly commuting self-maps of $M$ such that $T(M) \subset f(M)$. Suppose there exists $k \in(0,1)$ such that

$$
d(T x, T y) \leq k \max \{d(f x, f y), d(T x, f x), d(T y, f y), d(T x, f y), d(T y, f x)\}
$$

for all $x, y \in M$. If $\mathrm{cl}(T(M))$ is complete and $T$ is continuous, then there is a unique point $z$ in $M$ such that $T z=f z=z$. 
4 Common fixed point and approximations

We can prove now the following.

Theorem 2.2. Let $T$, I be self-maps on a subset $M$ of a p-normed space $X$. Assume that $M$ has the property $(N)$ with $q \in F(I)$, I satisfies the condition $(C)$ and $M=I(M)$. Suppose that $T$ and $I$ are $R$-subweakly commuting and satisfy

$$
\begin{gathered}
\|T x-T y\|_{p} \leq \max \left\{\|I x-I y\|_{p}, \operatorname{dist}(I x,[T x, q]), \operatorname{dist}(I y,[T y, q]),\right. \\
\operatorname{dist}(I x,[T y, q]), \operatorname{dist}(I y,[T x, q])\}
\end{gathered}
$$

for all $x, y \in M$. If $T$ is continuous, then $F(T) \cap F(I) \neq \varnothing$, provided one of the following conditions holds:

(i) $M$ is closed, $\mathrm{cl}(T(M))$ is compact and I is continuous,

(ii) $M$ is bounded and complete, $T$ is hemicompact and $I$ is continuous,

(iii) $M$ is bounded and complete, $T$ is condensing and $I$ is continuous,

(iv) $X$ is complete with separating dual $X^{*}, M$ is weakly compact, $T$ is completely continuous and $I$ is continuous.

Proof. Define $T_{n}$ by $T_{n} x=\left(1-k_{n}\right) q+k_{n} T x$ for all $x \in M$ and fixed sequence of real numbers $k_{n}\left(0<k_{n}<1\right)$ converging to 1 . Then, each $T_{n}$ is a well-defined self-mapping of $M$ as $M$ has property $(N)$ and for each $n, T_{n}(M) \subset M=I(M)$. Now the property $(C)$ of $I$ and the $R$-subweak commutativity of $\{T, I\}$ imply that

$$
\begin{aligned}
\left\|T_{n} I x-I T_{n} x\right\|_{p} & =\left(k_{n}\right)^{p}\|T I x-I T x\|_{p} \leq\left(k_{n}\right)^{p} R \operatorname{dist}(I x,[T x, q]) \\
& \leq\left(k_{n}\right)^{p} R\left\|T_{n} x-I x\right\|_{p}
\end{aligned}
$$

for all $x \in M$. This implies that the pair $\left\{T_{n}, I\right\}$ is $\left(k_{n}\right)^{p} R$-weakly commuting for each $n$. Also by (2.2),

$$
\begin{aligned}
\left\|T_{n} x-T_{n} y\right\|_{p}= & \left(k_{n}\right)^{p}\|T x-T y\|_{p} \\
\leq & \left(k_{n}\right)^{p} \max \left\{\|I x-I y\|_{p}, \operatorname{dist}(I x,[T x, q]), \operatorname{dist}(I y,[T y, q]),\right. \\
& \operatorname{dist}(I x,[T y, q]), \operatorname{dist}(I y,[T x, q])\} \\
\leq\left(k_{n}\right)^{p} \max \{ & \|I x-I y\|_{p},\left\|I x-T_{n} x\right\|_{p},\left\|I y-T_{n} y\right\|_{p}, \\
& \left.\left\|I x-T_{n} y\right\|_{p},\left\|I y-T_{n} x\right\|_{p}\right\}
\end{aligned}
$$

for each $x, y \in M$.

(i) Since $\mathrm{cl} T(M)$ is compact, $\mathrm{cl}\left(T_{n}(M)\right)$ is also compact. By Theorem 2.1, for each $n \geq 1$, there exists $x_{n} \in M$ such that $x_{n}=I x_{n}=T_{n} x_{n}$. The compactness of $\mathrm{cl} T(M)$ implies that there exists a subsequence $\left\{T x_{m}\right\}$ of $\left\{T x_{n}\right\}$ such that $T x_{m} \rightarrow y$ as $m \rightarrow \infty$. Then the definition of $T_{m} x_{m}$ implies $x_{m} \rightarrow y$, so by the continuity of $T$ and $I$ we have $y \in F(T) \cap$ $F(I)$. Thus $F(T) \cap F(I) \neq \varnothing$. 
(ii) As in (i) there exists $x_{n} \in M$ such that $x_{n}=I x_{n}=T_{n} x_{n}$. And $M$ is bounded, so $x_{n}-T x_{n}=\left(1-\left(k_{n}\right)^{-1}\right)\left(x_{n}-q\right) \rightarrow 0$ as $n \rightarrow \infty$ and hence $d_{p}\left(x_{n}, T x_{n}\right) \rightarrow 0$ as $n \rightarrow \infty$. The hemicompactness of $T$ implies that $\left\{x_{n}\right\}$ has a subsequence $\left\{x_{j}\right\}$ which converges to some $z \in M$. By the continuity of $T$ and $I$ we have $z \in F(T) \cap F(I)$. Thus $F(T) \cap F(I) \neq \varnothing$.

(iii) Every condensing map on a complete bounded subset of a metric space is hemicompact. Hence the result follows from (ii).

(iv) As in (i) there exists $x_{n} \in M$ such that $x_{n}=I x_{n}=T_{n} x_{n}$. Since $M$ is weakly compact, we can find a subsequence $\left\{x_{m}\right\}$ of $\left\{x_{n}\right\}$ in $M$ converging weakly to $y \in M$ as $m \rightarrow \infty$. Since $T$ is completely continuous, $T x_{m} \rightarrow T y$ as $m \rightarrow \infty$. Since $k_{n} \rightarrow 1, x_{m}=T_{m} x_{m}=$ $k_{m} T x_{m}+\left(1-k_{m}\right) q \rightarrow T y$ as $m \rightarrow \infty$. Thus $T x_{m} \rightarrow T^{2} y$ as $m \rightarrow \infty$ and consequently $T^{2} y=$ $T y$ implies that $T w=w$, where $w=T y$. Also, since $I x_{m}=x_{m} \rightarrow T y=w$, using the continuity of $I$ and the uniqueness of the limit, we have $I w=w$. Hence $F(T) \cap F(I) \neq \varnothing$.

It is clear that each $T$-invariant $q$-starshaped set satisfies the property $(N)$ and if $I$ is affine, then $I$ satisfies the condition $(C)$ and $T_{n}(M) \subset I(M)$ provided $T(M) \subset I(M)$ and $q \in F(I)$.

Corollary 2.3. Let $M$ be a closed $q$-starshaped subset of a p-normed space $X$, and $T$ and $I$ continuous self-maps of $M$. Suppose that $I$ is affine with $q \in F(I), T(M) \subset I(M)$ and $\mathrm{cl} T(M)$ is compact. If the pair $\{T, I\}$ is R-subweakly commuting and satisfy (2.2) for all $x, y \in M$, then $F(T) \cap F(I) \neq \varnothing$.

Corollary 2.4 [18, Theorem 2.2]. Let $M$ be a closed $q$-starshaped subset of a normed space $X$, and $T$ and I continuous self-maps of $M$. Suppose that $I$ is affine with $q \in F(I)$, $T(M) \subset I(M)$ and $\mathrm{cl} T(M)$ is compact. If the pair $\{T, I\}$ is $R$-subweakly commuting and satisfy, for all $x, y \in M$,

$$
\begin{gathered}
\|T x-T y\| \leq \max \{\|I x-I y\|, \operatorname{dist}(I x,[T x, q]), \operatorname{dist}(I y,[T y, q]), \\
\left.\frac{1}{2}[\operatorname{dist}(I x,[T y, q])+\operatorname{dist}(I y,[T x, q])]\right\},
\end{gathered}
$$

then $F(T) \cap F(I) \neq \varnothing$.

The following corollary improves and generalizes [1, Theorem 2.2].

Corollary 2.5. Let $M$ be a nonempty closed and $q$-starshaped subset of a p-normed space $X$ and $I$ be continuous self-map of $M$. Suppose that $I$ is affine with $q \in F(I), T(M) \subset$ $I(M)$ and $\mathrm{cl} T(M)$ is compact. If the pair $\{T, I\}$ is R-subweakly commuting and $T$ is $I$ nonexpansive on $M$, then $F(T) \cap F(I) \neq \varnothing$.

The following corollaries improve and generalize [3, Theorem 1] and [5, Theorem 4].

Corollary 2.6. Let $M$ be a nonempty closed and $q$-starshaped subset of a p-normed space $X, T$ and $I$ be continuous self-maps of $M$. Suppose that $I$ is affine with $q \in F(I), T(M) \subset$ $I(M)$ and $\mathrm{cl} T(M)$ is compact. If the pair $\{T, I\}$ is commuting and $T$ and I satisfy (2.2), then $F(T) \cap F(I) \neq \varnothing$. 
Corollary 2.7 [9, Theorem 2]. Let $M$ be a nonempty closed and q-starshaped subset of a $p$-normed space $X$. If $T$ is nonexpansive self-map of $M$ and $\operatorname{cl} T(M)$ is compact, then $F(T) \neq \varnothing$.

We now derive some approximation results.

Let $D_{M}^{R, I}(u)=P_{M}(u) \cap G_{M}^{R, I}(u)$, where $G_{M}^{R, I}(u)=\left\{x \in M:\|I x-u\|_{p} \leq(2 R+1) \operatorname{dist}(u, M)\right\}$.

The following result extends Theorem 2.3 of Shahzad [16] from the $I$-nonexpansiveness of $T$ to a more general condition.

Theorem 2.8. Let $M$ be subset of a p-normed space $X$ and $I, T: X \rightarrow X$ be mappings such that $u \in F(T) \cap F(I)$ for some $u \in X$ and $T(\partial M \cap M) \subset M$. If $I\left(D_{M}^{R, I}(u)\right)=D_{M}^{R, I}(u)$ and the pair $\{T, I\}$ is R-subweakly commuting and continuous on $D_{M}^{R, I}(u)$ and satisfy for all $x \in D_{M}^{R, I}(u) \cup\{u\}$,

$$
\|T x-T y\|_{p} \leq \begin{cases}\|I x-I u\|_{p} & \text { if } y=u, \\ \max \left\{\|I x-I y\|_{p}, \operatorname{dist}(I x,[q, T x]), \operatorname{dist}(I y,[q, T y]),\right. & \\ \operatorname{dist}(I x,[q, T y]), \operatorname{dist}(I y,[q, T x])\} & \text { if } y \in D_{M}^{R, I}(u),\end{cases}
$$

then $D_{M}^{R, I}(u)$ is T-invariant. Suppose that $D_{M}^{R, I}(u)$ is closed and $\operatorname{cl}\left(T\left(D_{M}^{R, I}(u)\right)\right)$ is compact. If $D_{M}^{R, I}(u)$ has property $(N)$ with $q \in F(I)$, and I satisfies property $(C)$ on $D_{M}^{R, I}(u)$, then $P_{M}(u) \cap F(I) \cap F(T) \neq \varnothing$.

Proof. Let $x \in D_{M}^{R, I}(u)$. Then, $x \in P_{M}(u)$ and hence $\|x-u\|_{p}=\operatorname{dist}(u, M)$. Note that for any $k \in(0,1)$,

$$
\|k u+(1-k) x-u\|_{p}=(1-k)^{p}\|x-u\|_{p}<\operatorname{dist}(u, M) .
$$

It follows that the line segment $\{k u+(1-k) x: 0<k<1\}$ and the set $M$ are disjoint. Thus $x$ is not in the interior of $M$ and so $x \in \partial M \cap M$. Since $T(\partial M \cap M) \subset M$, Tx must be in $M$. Also since $I x \in P_{M}(u), u \in F(T) \cap F(I)$ and $T$ and $I$ satisfy (2.6), we have

$$
\|T x-u\|_{p}=\|T x-T u\|_{p} \leq\|I x-I u\|_{p}=\|I x-u\|_{p}=\operatorname{dist}(u, M) .
$$

Thus $T x \in P_{M}(u)$. From the $R$-subweak commutativity of the pair $\{T, I\}$ and (2.6), it follows that (see also proof of [16, Theorem 2.3]),

$$
\begin{aligned}
\|I T x-u\|_{p} & =\|I T x-T I x+T I x-T u\|_{p} \leq R\|T x-I x\|_{p}+\left\|I^{2} x-I u\right\|_{p} \\
& =R\|T x-u+u-I x\|_{p}+\left\|I^{2} x-u\right\|_{p} \\
& \leq R\left(\|T x-u\|_{p}+\|I x-u\|_{p}\right)+\left\|I^{2} x-u\right\|_{p} \\
& \leq(2 R+1) \operatorname{dist}(u, M) .
\end{aligned}
$$

Thus $T x \in G_{M}^{R, I}(u)$. Consequently, $T\left(D_{M}^{R, I}(u)\right) \subset D_{M}^{R, I}(u)=I\left(D_{M}^{R, I}(u)\right)$. Now Theorem 2.2(i) guarantees that, $P_{M}(u) \cap F(I) \cap F(T) \neq \varnothing$. 
Remarks 2.9. (1) If $p=1$ and $M$ is $q$-starshaped with $q \in F(I), T(M) \subset I(M)$ and $I$ is linear on $D_{M}^{R, I}(u)$ in Theorem 2.8, we obtain the conclusion of a recent result [18, Theorem 2.5] for the more general inequality (2.6).

(2) Let $C_{M}^{I}(u)=\left\{x \in M: I x \in P_{M}(u)\right\}$. Then $I\left(P_{M}(u)\right) \subset P_{M}(u)$ implies $P_{M}(u) \subset$ $C_{M}^{I}(u) \subset G_{M}^{R, I}(u)$ and hence $D_{M}^{R, I}(u)=P_{M}(u)$. Consequently, Theorem 2.8 remains valid when $D_{M}^{R, I}(u)=P_{M}(u)$. Hence we obtain the following result which contains properly Theorems 1.2 and 1.3 and improves and extends Theorem 8 of [5], Theorem 4 in [9], and Theorem 6 in $[14,15]$.

Corollary 2.10. Let $M$ be subset of a $p$-normed space $X$ and let $I, T: X \rightarrow X$ be mappings such that $u \in F(T) \cap F(I)$ for some $u \in X$ and $T(\partial M \cap M) \subset M$. Assume that $I\left(P_{M}(u)\right)=$ $P_{M}(u)$ and the pair $\{T, I\}$ is $R$-subweakly commuting and continuous on $P_{M}(u)$ and satisfy for all $x \in P_{M}(u) \cup\{u\}$,

$$
\|T x-T y\|_{p} \leq \begin{cases}\|I x-I u\|_{p} & \text { if } y=u, \\ \max \left\{\|I x-I y\|_{p}, \operatorname{dist}(I x,[q, T x]), \operatorname{dist}(I y,[q, T y]),\right. & \\ \operatorname{dist}(I x,[q, T y]), \operatorname{dist}(I y,[q, T x])\} & \text { if } y \in P_{M}(u) .\end{cases}
$$

Suppose that $P_{M}(u)$ is closed, q-starshaped with $q \in F(I), I$ is affine and $\operatorname{cl}\left(T\left(P_{M}(u)\right)\right)$ is compact. Then $P_{M}(u) \cap F(I) \cap F(T) \neq \varnothing$.

Let $D=P_{M}(u) \cap C_{M}^{I}(u)$, where $C_{M}^{I}(u)=\left\{x \in M: I x \in P_{M}(u)\right\}$.

The following result contains Theorem 1.4 and many others.

Theorem 2.11. Let $M$ be subset of a p-normed space $X$ and $I, T: X \rightarrow X$ be mappings such that $u \in F(T) \cap F(I)$ for some $u \in X$ and $T(\partial M \cap M) \subset M$. If $I(D)=D$ and the pair $\{T, I\}$ is commuting and continuous on $D$ and satisfy for all $x \in D \cup\{u\}$,

$$
\|T x-T y\|_{p} \leq \begin{cases}\|I x-I u\|_{p} & \text { if } y=u, \\ \max \left\{\|I x-I y\|_{p}, \operatorname{dist}(I x,[q, T x]), \operatorname{dist}(I y,[q, T y]),\right. & \\ \operatorname{dist}(I x,[q, T y]), \operatorname{dist}(I y,[q, T x])\} & \text { if } y \in D,\end{cases}
$$

then $D$ is T-invariant. Suppose that $D$ is closed and $\mathrm{cl}(T(D))$ is compact. If $D$ has property (N) with $q \in F(I)$, and I satisfies property $(C)$ on $D$, then $P_{M}(u) \cap F(I) \cap F(T) \neq \varnothing$.

Proof. Let $x \in D$, then proceeding as in the proof of Theorem 2.8, we obtain $T x \in P_{M}(u)$. Moreover, since $T$ commutes with $I$ on $D$ and $T$ satisfies (2.11),

$$
\|I T x-u\|_{p}=\|T I x-T u\|_{p} \leq\left\|I^{2} x-I u\right\|_{p}=\left\|I^{2} x-u\right\|_{p}=\operatorname{dist}(u, M) .
$$

Thus IT $x \in P_{M}(u)$ and so $T x \in C_{M}^{I}(u)$. Hence $T x \in D$. Consequently, $T(D) \subset D=I(D)$. Now Theorem 2.2(i) guarantees that $P_{M}(u) \cap F(I) \cap F(T) \neq \varnothing$.

In the following result we obtain a non-locally convex space analogue of [6, Theorem 3.3] for nonstarshaped set $D$. 
Theorem 2.12. Let $M$ be subset of a p-normed space $X$ and $I, T: X \rightarrow X$ be mappings such that $u \in F(T) \cap F(I)$ for some $u \in X$ and $T(\partial M \cap M) \subset M$. If $I(D)=D$ and the pair $\{T, I\}$ is $R$-subweakly commuting and continuous on $D$ and, for all $x \in D \cup\{u\}$, satisfies the following inequality,

$$
\|T x-T y\|_{p} \leq \begin{cases}\|I x-I u\|_{p} & \text { if } y=u, \\ \max \left\{\|I x-I y\|_{p}, \operatorname{dist}(I x,[q, T x]), \operatorname{dist}(I y,[q, T y]),\right. & \\ \operatorname{dist}(I x,[q, T y]), \operatorname{dist}(I y,[q, T x])\} & \text { if } y \in D,\end{cases}
$$

and $I$ is nonexpansive on $P_{M}(u) \cup\{u\}$, then $D$ is T-invariant. Suppose that $D$ is closed, has property $(N)$ with $q \in F(I), \operatorname{cl}(T(D))$ is compact and I satisfies property $(C)$ on $D$. Then $P_{M}(u) \cap F(I) \cap F(T) \neq \varnothing$.

Proof. Let $x \in D$, then proceeding as in the proof of Theorem 2.8, we obtain Tx $\in P_{M}(u)$. Moreover, since $I$ is nonexpansive on $P_{M}(u) \cup\{u\}$ and $T$ satisfies (2.13), we obtain

$$
\|I T x-u\|_{p} \leq\|T x-T u\|_{p} \leq\|I x-I u\|_{p}=\operatorname{dist}(u, M)
$$

Thus $I T x \in P_{M}(u)$ and so $T x \in C_{M}^{I}(u)$. Hence $T x \in D$. Consequently, $T(D) \subset D=I(D)$. Now Theorem 2.2(i) guarantees that $P_{M}(u) \cap F(I) \cap F(T) \neq \varnothing$.

Remark 2.13. Notice that approximation results similar to Theorems 2.8, 2.11, and 2.12 can be obtained, using Theorem 2.2(ii), (iii), and (iv).

Example 2.14. Let $X=R$ and $M=\{0,1,1-1 /(n+1): n \in N\}$ be endowed with usual metric. Define $T 1=0$ and $T 0=T(1-1 /(n+1))=1$ for all $n \in N$. Clearly, $M$ is not starshaped but $M$ has the property $(N)$ for $q=0$ and $k_{n}=1-1 /(n+1), n \in N$. Let $I x=x$ for all $x \in M$. Now $I$ and $T$ satisfy (2.2) together with all other conditions of Theorem 2.2(i) except the condition that $T$ is continuous. Note that $F(I) \cap F(T)=\varnothing$.

Example 2.15. Let $X=R^{2}$ be endowed with the $p$-norm $\|,\|_{p}$ defined by $\|(a, b)\|_{p}=$ $|a|^{p}+|b|^{p},(a, b) \in R^{2}$.

(1) Let $M=A \cup B$, where $A=\{(a, b) \in X: 0 \leq a \leq 1,0 \leq b \leq 4\}$ and $B=\{(a, b) \in X$ : $2 \leq a \leq 3,0 \leq b \leq 4\}$. Define $T: M \rightarrow M$ by

$$
T(a, b)= \begin{cases}(2, b) & \text { if }(a, b) \in A \\ (1, b) & \text { if }(a, b) \in B\end{cases}
$$

and $I(x)=x$, for all $x \in M$. All of the conditions of Theorem 2.2(i) are satisfied except that $M$ has property $(N)$, that is, $\left(1-k_{n}\right) q+k_{n} T(M)$ is not contained in $M$ for any choice of $q \in M$ and $k_{n}$. Note $F(I) \cap F(T)=\varnothing$. 
(2) If $M=\{(a, b) \in X: 0 \leq a<\infty, 0 \leq b \leq 1\}$ and $T: M \rightarrow M$ is defined by

$$
T(a, b)=(a+1, b), \quad(a, b) \in M
$$

Define $I(x)=x$, for all $x \in M$. All of the conditions of Theorem 2.2(i) are satisfied except that $M$ is compact. Note $F(I) \cap F(T)=\varnothing$. Notice that $M$, being convex and $T$-invariant, has the property $(N)$ for any choice of $q$ and $\left\{k_{n}\right\}$.

(3) If $M=\{(a, b) \in X: 0<a<1,0<b<1\}$ and $T, I: M \rightarrow M$ are defined by $T(a, b)=$ $(a / 2, b / 3)$, and $I(x)=x$ for all $x \in M$. All of the conditions of Theorem 2.2(i) are satisfied except the fact that $M$ is closed. However $F(I) \cap F(T)=\varnothing$.

Example 2.16. Let $X=R$ and $M=[0,1]$ be endowed with the usual metric. Define $T(x)=$ 0 and $I(x)=1-x$ for each $x \in M$. All of the conditions of Theorem 2.2(i) are satisfied except the condition that the pair $\{I, T\}$ is $R$-subweakly commuting. Note $F(I) \cap F(T)=$ $\varnothing$.

\section{Further results}

All results of the paper (Theorem 2.2-Remark 2.13) remain valid in the setup of a metrizable locally convex topological vector space(tvs) $(X, d)$ where $d$ is translation invariant and $d(\alpha x, \alpha y) \leq \alpha d(x, y)$, for each $\alpha$ with $0<\alpha<1$ and $x, y \in X$ (recall that $d_{p}$ is translation invariant and satisfies $d_{p}(\alpha x, \alpha y) \leq \alpha^{p} d_{p}(x, y)$ for any scalar $\left.\alpha \geq 0\right)$. Consequently, Theorem 2.2 (i)-(ii) and Theorem 3.3 (i)-(ii) due to Hussain and Khan [6] and Theorem 3.5 (i)-(ii) \& (v), (ix)-(x) and Theorem 4.2 (i)-(ii) \& (v), (ix)-(x) due to Hussain et al. [7] are extended to a class of maps satisfying a more general inequality.

From Corollary 2.3, we have the following result which extends [18, Theorem 2.2];

COROLlARY 3.1. Let $M$ be a closed $q$-starshaped subset of a metrizable locally convex space $(X, d)$ where $d$ is translation invariant and $d(\alpha x, \alpha y) \leq \alpha d(x, y)$, for each $\alpha$ with $0<\alpha<1$ and $x, y \in X$. Suppose that $T$ and I are continuous self-maps of $M, I$ is affine with $q \in F(I)$, $T(M) \subset I(M)$ and $\mathrm{cl} T(M)$ is compact. If the pair $\{T, I\}$ is $R$-subweakly commuting and satisfy for all $x, y \in M$,

$$
\begin{gathered}
d(T x, T y) \leq \max \{d(I x, I y), \operatorname{dist}(I x,[T x, q]), \operatorname{dist}(I y,[T y, q]), \\
\operatorname{dist}(I x,[T y, q]), \operatorname{dist}(I y,[T x, q])\}
\end{gathered}
$$

then $F(T) \cap F(I) \neq \varnothing$.

We define $C_{M}^{I}(u)=\left\{x \in M: I x \in P_{M}(u)\right\}$ and denote by $\mathfrak{J}_{0}$ the class of closed convex subsets of $X$ containing 0 . For $M \in \mathfrak{J}_{0}$, we define $M_{u}=\{x \in M:\|x\| \leq 2\|u\|\}$. It is clear that $P_{M}(u) \subset M_{u} \in \mathfrak{J}_{0}$.

Following result includes [1, Theorem 4.1] and [5, Theorem 8$]$ and provides an analogue of [18, Theorem 2.8] in the setting of metrizable locally convex space and contractive condition involved is more general.

Theorem 3.2. Let $X$ be as in Corollary 3.1, and $T$ be a self-mapping of $X$ with $u \in F(T)$, $M \in \mathfrak{J}_{0}$ such that $T(M) \subset M$. Suppose that $\mathrm{cl} T(M)$ is compact, $T$ is continuous on $M$ and 
satisfies for all $x \in M \cup\{u\}$,

$$
d(T x, T y) \leq \begin{cases}d(x, u) & \text { if } y=u, \\ \max \{d(x, y), \operatorname{dist}(x,[0, T x]), \operatorname{dist}(y,[0, T y]), & \\ \operatorname{dist}(x,[0, T y]), \operatorname{dist}(y,[0, T x])\} & \text { if } y \in M,\end{cases}
$$

then

(i) $P_{M}(u)$ is nonempty, closed, and convex,

(ii) $T\left(P_{M}(u)\right) \subset P_{M}(u)$,

(iii) $P_{M}(u) \cap F(T) \neq \varnothing$.

Proof. (i) Let $r=\operatorname{dist}(u, M)$. Then there is a minimizing sequence $\left\{y_{n}\right\}$ in $M$ such that $\lim _{n} d\left(u, y_{n}\right)=r$. As $\operatorname{cl} T(M)$ is compact so $\left\{T y_{n}\right\}$ has a convergent subsequence $\left\{T y_{m}\right\}$ with $\lim T y_{m}=x_{0}$ (say) in $M$. Now by (3.2)

$$
r \leq d\left(x_{0}, u\right)=\lim d\left(T y_{m}, u\right) \leq \lim d\left(y_{m}, u\right)=\lim d\left(y_{n}, u\right)=r .
$$

Hence $x_{0} \in P_{M}(u)$. Thus $P_{M}(u)$ is nonempty closed and convex.

(ii) Let $z \in P_{M}(u)$. Then $d(T z, u)=d(T z, T u) \leq d(z, u)=\operatorname{dist}(u, M)$. This implies that $T z \in P_{M}(u)$ and so $T\left(P_{M}(u)\right) \subset P_{M}(u)$.

(iii) As $\mathrm{cl} T\left(P_{M}(u)\right) \subset \mathrm{cl} T(M)$, so $\mathrm{cl} T\left(P_{M}(u)\right)$ is compact. Thus by Corollary 3.1, $P_{M}(u) \cap F(T) \neq \varnothing$.

Theorem 3.3. Let $X$ be as in Theorem 3.2 and $I$ and $T$ be self-mappings of $X$ with $u \in$ $F(I) \cap F(T)$ and $M \in \mathfrak{I}_{0}$ such that $T\left(M_{u}\right) \subset I(M) \subset M$. Suppose that $I$ is affine and continuous on $M, d(I x, u) \leq d(x, u)$ for all $x \in M, \operatorname{cl} I(M)$ is compact and I satisfies for all $x, y \in M$,

$$
\begin{gathered}
d(I x, I y) \leq \max \{d(x, y), \operatorname{dist}(x,[0, I x]), \operatorname{dist}(y,[0, I y]), \\
\operatorname{dist}(x,[0, I y]), \operatorname{dist}(y,[0, I x])\} .
\end{gathered}
$$

If the pair $\{T, I\}$ is R-subweakly commuting and $T$ is continuous on $M_{u}$ and satisfy for all $x, y \in M_{u} \cup\{u\}$, and $q \in F(I)$,

$$
d(T x, T y) \leq \begin{cases}d(I x, I u) & \text { if } y=u, \\ \max \{d(I x, I y), \operatorname{dist}(I x,[q, T x]), \operatorname{dist}(I y,[q, T y]), & \\ \operatorname{dist}(I x,[q, T y]), \operatorname{dist}(I y,[q, T x])\} & \text { if } y \in M_{u},\end{cases}
$$

then

(i) $P_{M}(u)$ is nonempty, closed, and convex,

(ii) $T\left(P_{M}(u)\right) \subset I\left(P_{M}(u)\right) \subset P_{M}(u)$,

(iii) $P_{M}(u) \cap F(I) \cap F(T) \neq \varnothing$.

Proof. From Theorem 3.2, we obtain (i). Also we have $I\left(P_{M}(u)\right) \subset P_{M}(u)$. Let $y \in$ $T P_{M}(u)$. Since $T\left(M_{u}\right) \subset I(M)$ and $P_{M}(u) \subset M_{u}$, there exist $z \in P_{M}(u)$ and $x \in M$ such 
that $y=T z=I x$. By (3.5), we have

$$
d(I x, u)=d(T z, T u) \leq d(I z, I u) \leq d(z, u)=\operatorname{dist}(u, M) .
$$

Hence $x \in C_{M}^{I}(u)=P_{M}(u)$ and so (ii) holds.

(iii) Theorem 3.2 guarantees that $P_{M}(u) \cap F(I) \neq \varnothing$. Thus there exists $q \in P_{M}(u)$ such that $q \in F(I)$. Hence the conclusion follows from Corollary 3.1.

Following corollary provides the conclusions of [1, Theorem 4.2(a)] and [17, Theorem 2.3 ], to the setup of metrizable locally convex space.

Corollary 3.4. Let $X$ be as above and $I, T$ be self-mappings of $X$ with $u \in F(I) \cap F(T)$ and $M \in \mathfrak{J}_{0}$ such that $T\left(M_{u}\right) \subset I(M) \subset M$. Suppose that $I$ is affine and continuous on $M$, $d(I x, u) \leq d(x, u)$ for all $x \in M, \operatorname{cl} I(M)$ is compact and $I$ is nonexpansive on $M$. If the pair $\{T, I\}$ is $R$-subweakly commuting on $M_{u}$ and $T$ is I-nonexpansive on $M_{u} \cup\{u\}$, then

(i) $P_{M}(u)$ is nonempty, closed and convex,

(ii) $T\left(P_{M}(u)\right) \subset I\left(P_{M}(u)\right) \subset P_{M}(u)$,

(iii) $P_{M}(u) \cap F(I) \cap F(T) \neq \varnothing$.

Let $(X, d)$ be a metric linear space with translation invariant metric $d$. We say that the metric $d$ is strictly monotone [4], if $x \neq 0$ and $0<t<1$ imply $d(0, t x)<d(0, x)$. Each $p$-norm generates a translation invariant metric, which is strictly monotone [4].

Following the arguments of Jungck [8, Theorem 3.2] and using Theorem 2.1 instead of Theorem 3.1 of Jungck [8], we obtain,

Theorem 3.5. Let $T$ and $f$ be continuous self-maps of a compact metric space $(X, d)$ with $T(X) \subset f(X)$. If $T$ and $f$ are $R$-weakly commuting self-maps of $X$ such that

$$
d(T x, T y)<\max \{d(f x, f y), d(T x, f x), d(T y, f y), d(T x, f y), d(T y, f x)\}
$$

when right hand side is positive, then there is a unique point $z$ in $X$ such that $T z=f z=z$.

Using Theorem 3.5, we establish common fixed point generalization of Theorem 1 of Dotson [3], and Theorem 2 of Guseman and Peters [4].

Theorem 3.6. Let $T$, I be self-maps on a compact subset $M$ of a metric linear space $(X, d)$ with translation invariant and strictly monotone metric $d$. Assume that $M$ has the property $(N)$ with $q \in F(I)$, I satisfies the condition $(C)$ and $M=I(M)$. Suppose that $T$ and $I$ are $R$-subweakly commuting and satisfy

$$
\begin{gathered}
d(T x, T y) \leq \max \{d(I x, I y), \operatorname{dist}(I x,[T x, q]), \operatorname{dist}(I y,[T y, q]), \\
\operatorname{dist}(I x,[T y, q]), \operatorname{dist}(I y,[T x, q])\}
\end{gathered}
$$

for all $x, y \in M$. If $T$ and $I$ are continuous, then $F(T) \cap F(I) \neq \varnothing$.

Proof. Proof is similar to Theorem 2.2(i), instead of applying Theorem 2.1, we apply Theorem 3.5. 
Similarly, all other results of Section 2 (Corollary 2.3-Theorem 2.12) hold in the setting of metric linear space $(X, d)$ with translation invariant and strictly monotone metric $d$ provided we replace closedness of $M$ and compactness of $\mathrm{cl} T(M)$ by compactness of $M$ and using Theorem 3.6 instead of Theorem 2.2(i). Consequently, metric linear space versions of Corollary 2.3-Corollary 2.7 improve and extend Theorem 2 and the Corollary in [4].

A metric space $(X, d)$ is said to be $S$-space [20], if there exists an $x_{0}$ in $X$ such that for every $t \in(0,1)$ there is a $d$-contractive self-mapping $f_{t}$ of $X$ for which the inequality $d\left(f_{t}(x), x\right) \leq(1-t) d\left(x_{0}, x\right)$ holds for every $x$ in $X$. As an application of Theorem 3.5 and [20, Theorem 1], we obtain the following extension of Theorems $B, K, Z$ and $C$ in [2] and Theorem 3 of [20] to generalized nonexpansive mappings.

Theorem 3.7. Let $(X, d)$ be a compact $S$-space and $T: X \rightarrow X$ satisfies for all $x, y \in X$,

$$
d(T x, T y) \leq \max \{d(x, y), d(x, T x), d(y, T y), d(x, T y), d(y, T x)\} .
$$

Then T has a fixed point.

\section{Acknowledgment}

The authors are grateful to N. Shahzad for providing preprint of [18].

\section{References}

[1] M. A. Al-Thagafi, Common fixed points and best approximation, Journal of Approximation Theory 85 (1996), no. 3, 318-323.

[2] V. Berinde, A common fixed point theorem for quasi contractive type mappings, Annales Universitatis Scientiarum Budapestinensis de Rolando Eötvös Nominatae. Sectio Mathematica 46 (2003), 101-110 (2004).

[3] W. G. Dotson Jr., Fixed point theorems for non-expansive mappings on star-shaped subsets of Banach spaces, Journal of the London Mathematical Society. Second Series 4 (1971/1972), 408410.

[4] L. F. Guseman Jr. and B. C. Peters Jr., Nonexpansive mappings on compact subsets of metric linear spaces, Proceedings of the American Mathematical Society 47 (1975), 383-386.

[5] L. Habiniak, Fixed point theorems and invariant approximations, Journal of Approximation Theory 56 (1989), no. 3, 241-244.

[6] N. Hussain and A. R. Khan, Common fixed-point results in best approximation theory, Applied Mathematics Letters. An International Journal of Rapid Publication 16 (2003), no. 4, 575-580.

[7] N. Hussain, D. O'Regan, and R. P. Agarwal, Common fixed point and invariant approximation results on non-starshaped domain, Georgian Mathematical Journal 12 (2005), no. 4, 559-669.

[8] G. Jungck, Common fixed points for commuting and compatible maps on compacta, Proceedings of the American Mathematical Society 103 (1988), no. 3, 977-983.

[9] L. A. Khan and A. R. Khan, An extension of Brosowski-Meinardus theorem on invariant approximation, Approximation Theory and its Applications. New Series 11 (1995), no. 4, 1-5.

[10] G. Meinardus, Invarianz bei linearen Approximationen, Archive for Rational Mechanics and Analysis 14 (1963), 301-303 (German).

[11] S. A. Naimpally, K. L. Singh, and J. H. M. Whitfield, Fixed points and nonexpansive retracts in locally convex spaces, Polska Akademia Nauk. Fundamenta Mathematicae 120 (1984), no. 1, 6375 . 
[12] W. Rudin, Functional Analysis, 2nd ed., International Series in Pure and Applied Mathematics, McGraw-Hill, New York, 1991.

[13] S. A. Sahab, M. S. Khan, and S. Sessa, A result in best approximation theory, Journal of Approximation Theory 55 (1988), no. 3, 349-351.

[14] N. Shahzad, A result on best approximation, Tamkang Journal of Mathematics 29 (1998), no. 3, 223-226.

[15] Correction to: "A result on best approximation", Tamkang Journal of Mathematics 30 (1999), no. 2, 165.

[16] Noncommuting maps and best approximations, Radovi Matematički 10 (2000/2001), no. $1,77-83$.

[17] _ Invariant approximations and R-subweakly commuting maps, Journal of Mathematical Analysis and Applications 257 (2001), no. 1, 39-45.

[18] _ Invariant approximations, generalized I-contractions, and R-subweakly commuting maps, Fixed Point Theory and Applications 2005 (2005), no. 1, 79-86.

[19] S. P. Singh, An application of a fixed-point theorem to approximation theory, Journal of Approximation Theory 25 (1979), no. 1, 89-90.

[20] L. A. Talman, A fixed point criterion for compact $T_{2}$-spaces, Proceedings of the American Mathematical Society 51 (1975), 91-93.

Nawab Hussain: Centre for Advanced Studies in Pure Applied Mathematics,

Bahauddin Zakariya University, Multan, Pakistan

Current address: Department of Mathematics, Faculty of Science, King Abdul Aziz University,

P.O. Box 80203, Jeddah 21589, Saudi Arabia

E-mail address: mnawab2000@yahoo.com

Vasile Berinde: Department of Mathematics and Computer Science, Faculty of Sciences, North University of Baia Mare, Victoriei Nr. 76, 430122 Baia Mare, Romania

E-mail address: vberinde@ubm.ro 